\title{
Elimination of Disjunction and Negation in Answer-Set Programs under Hyperequivalence ${ }^{\star}$
}

\author{
Jörg Pührer, Hans Tompits, and Stefan Woltran \\ Institut für Informationssysteme, Technische Universität Wien, \\ Favoritenstraße 9-11, A-1040 Vienna, Austria \\ \{puehrer, tompits\}@kr.tuwien.ac.at \\ woltrandabai.tuwien.ac.at
}

\begin{abstract}
The study of different notions of equivalence is one of the cornerstones of current research in answer-set programming. This is mainly motivated by the needs of program simplification and modular programming, for which ordinary equivalence is insufficient. A recently introduced equivalence notion in this context is hyperequivalence, which includes as special cases strong, uniform, and ordinary equivalence. We study in this paper the question of replacing programs by syntactically simpler ones preserving hyperequivalence (we refer to such a replacement as a casting). In particular, we provide necessary and sufficient semantic conditions under which the elimination of disjunction, negation, or both, in programs is possible, preserving hyperequivalence. In other words, we characterise in model-theoretic terms when a disjunctive logic program can be replaced by a hyperequivalent normal, positive, or Horn program, respectively. Furthermore, we study the computational complexity of the considered tasks and, based on similar results for strong equivalence developed in previous work, we provide methods for constructing the respective hyperequivalent programs. Our results contribute to the understanding of problem settings in logic programming in the sense that they show in which scenarios the usage of certain constructs are superfluous or not.
\end{abstract}

\section{Introduction}

Answer-set programming (ASP) is an important logic-programming paradigm [1] that is based on principles of nonmonotonic reasoning and became popular for its fully declarative semantics [2]. An important research field in ASP is the study of equivalence of answer-set programs. Given the nonmonotonic nature of logic programs under the answer-set semantics, ordinary equivalence (which holds between two programs if their answer sets coincide) is too weak to yield a replacement property similar to the one of classical logic. That is to say, given a program $P=Q \cup R$, when replacing $Q$ with an ordinarily equivalent program $Q^{\prime}$, it is not guaranteed that $Q^{\prime} \cup R$ is ordinarily equivalent to $P$. This led to the introduction of stricter notions of equivalence, in particular strong and uniform equivalence: two programs, $P$ and $Q$, are strongly equivalent [3] if $P \cup R$ and $Q \cup R$ have the same answer sets for any program $R$, called the context, while

\footnotetext{
* This work was partially supported by the Austrian Science Fund (FWF) under projects P18019
} and P20704. 
they are uniformly equivalent [4] if the context is restricted to sets of facts. Recently, Woltran [5] introduced hyperequivalence, or head-body relativised equivalence, which is a parametrised notion that subsumes as special cases strong, uniform, and ordinary equivalence. It allows for specifying, on the one hand, the atoms which are permitted to occur in the rule heads of context programs and, on the other hand, the atoms allowed in the rule bodies. Besides generalising various equivalence notions, hyperequivalence can be parametrised for application-specific equivalence tests [6].

In this paper, we are interested in the question whether a given disjunctive logic program $P$ can be replaced by a program $Q$ that is from a syntactically simpler program class than $P$ preserving hyperequivalence (we refer to $Q$ as a casting of $P$ ). In particular, we are interested in the questions whether a given program can be casted (i) to a program without disjunctions, (ii) to a program without negations, and (iii) to a program without both disjunctions and negations. There is previous work addressing these questions for the notions of strong and uniform equivalence [7,8], introducing modeltheoretic characterisations when a casting is possible. We will introduce such conditions for the general case of hyperequivalence, and thereby obtain proper generalisations of the old concepts. More specifically, our main contributions are the following:

- We introduce necessary and sufficient conditions for deciding whether, for a given program, a hyperequivalent normal, positive, or Horn program exists. These conditions are model-theoretic, operating on sets of SE-interpretations [9], which are well-known structures derived from the logical underpinning of strong equivalence [3].

- We provide methods that allow the construction of a casting, whenever a given program is castable. That is, if a program satisfies one of our model-theoretic conditions, we give a constructive method for finding a desired hyperequivalent program.

- We analyse the complexity of the casting problems under consideration. It turns out that these are located on the second and third level of the polynomial hierarchy.

In many situations, our results allow for program simplifications that are not possible under stronger notions of equivalence. For example, the program $P_{\mathrm{ex}}=\{f \leftarrow$ $b$, not $n ; n \leftarrow p ; b \vee p\}$ cannot be replaced by a program $Q$ without negations such that $P_{\text {ex }} \cup R$ and $Q \cup R$ have the same answer sets for every program $R$. However, such a $Q$ exists whenever atoms $b$ and $n$ do not occur in the head of any rule of $R$.

Casting under hyperequivalence is also essential for program simplification and modular programming: Depending on the atoms permitted to occur in the rule heads and rule bodies in the context, a module can faithfully be replaced by a simpler one. Moreover, understanding under which circumstances such a replacement is possible gives insight into which roles negation and disjunction play in a certain program.

\section{Preliminaries}

We deal with finite propositional disjunctive logic programs containing rules (over a set At of atoms) of form $a_{1} \vee \cdots \vee a_{l} \leftarrow b_{1}, \ldots, b_{m}$, not $b_{m+1}, \ldots$, not $b_{n}$, where $l \geq 0$, $n \geq m \geq 0$, all $a_{i}, b_{j}$ are from $A t$, and not denotes default negation. A rule $r$ as described is normal, if $l \leq 1$; positive, if $m=n$; and a fact, if $l=1$ and $m=n=0$. 
A rule is Horn if it is positive and normal. The head of $r$ is the set $H(r)=\left\{a_{1}, \ldots, a_{l}\right\}$; the body of $r$ is $B(r)=\left\{b_{1}, \ldots, b_{m}\right.$, not $b_{m+1}, \ldots$, not $\left.b_{n}\right\}$. We also define $B^{+}(r)=$ $\left\{b_{1}, \ldots, b_{m}\right\}$ and $B^{-}(r)=\left\{b_{m+1}, \ldots, b_{n}\right\}$. A disjunctive logic program (DLP) over $A t$, or simply a program, is a finite set of rules over At. A DLP $P$ is a normal logic program (NLP) if every rule in $P$ is normal. Likewise, $P$ is a positive logic program (PLP) if every rule in $P$ is normal, and it is a Horn program if every rule in it is Horn. We denote the class of all DLPs (resp., NLPs, PLPs, Horn programs) by $\mathcal{D} \mathcal{L P}$ (resp., $\mathcal{N} \mathcal{L} \mathcal{P}, \mathcal{P} \mathcal{L} \mathcal{P}, \mathcal{H} \mathcal{O} \mathcal{N} \mathcal{N})$. Furthermore, $\operatorname{atm}(P)$ stands for the set of all atoms occurring in $P$, and we define $H(P)=\bigcup_{r \in P} H(r)$ and $B(P)=\bigcup_{r \in P}\left(B^{+}(r) \cup B^{-}(r)\right)$.

Let $I$ be an interpretation, i.e., a set of atoms. $I$ satisfies a rule $r$, symbolically $I \models r$, iff $I \cap H(r) \neq \emptyset$ whenever $B^{+}(r) \subseteq I$ and $I \cap B^{-}(r)=\emptyset$ jointly hold. Furthermore, $I$ is a model of a program $P$, symbolically $I \models P$, iff $I \models r$, for all $r \in P . I$ is an answer set [2] of a program $P$ iff $I$ is a minimal model of $P^{I}$, where $P^{I}=\left\{H(r) \leftarrow B^{+}(r) \mid r \in P, B^{-}(r) \cap I=\emptyset\right\}$ is the reduct of $P$ relative to $I$.

We recall the recently introduced notion of hyperequivalence, also called headbody-relativised equivalence [5]. The idea is to restrict the alphabets of rule heads and rule bodies of the context programs, and thereby limiting the nonmonotonic side-effects of putting a program in some context. For alphabets $\mathcal{H}, \mathcal{B} \subseteq A t$, let $\mathcal{D} \mathcal{L} \mathcal{P}_{\mathcal{B}}^{\mathcal{H}}$ denote the class of all programs $P$ such that $H(P) \subseteq \mathcal{H}$ and $B(P) \subseteq \mathcal{B}$. Then, two programs $P, Q$ over $A t$ are hyperequivalent relative to $\langle\mathcal{H}, \mathcal{B}\rangle$, or $\langle\mathcal{H}, \mathcal{B}\rangle$-equivalent, in symbols $P \equiv_{\mathcal{B}}^{\mathcal{H}} Q$, iff, for each $R \in \mathcal{D} \mathcal{L} \mathcal{P}_{\mathcal{B}}^{\mathcal{H}}$, it holds that $\mathcal{A} \mathcal{S}(P \cup R)=\mathcal{A S}(Q \cup R)$. Hyperequivalence includes well-known equivalence notions as special cases: strong equivalence [3] coincides with $\langle A t, A t\rangle$-equivalence; uniform equivalence [4] coincides with $\langle A t, \emptyset\rangle$-equivalence; and ordinary equivalence coincides with $\langle\emptyset, \emptyset\rangle$-equivalence.

Following Turner [9], strong equivalence can model-theoretically be characterised in terms of SE-models: First of all, by an SE-interpretation we understand a pair $(X, Y)$, where $X, Y \subseteq A t$ are sets of atoms such that $X \subseteq Y$. If $X=Y$, then $(X, Y)$ is total, otherwise $(X, Y)$ is non-total. An SE-interpretation $(X, Y)$ is an SE-model of a program $P$ over $A t$ if $Y \models P$ and $X \models P^{Y}$. It then holds that two programs $P$ and $Q$ are strongly equivalent iff they possess the same set of SE-models. In view of the logical underpinning of strong equivalence [3], viz., the logic of here-and-there [10] (also known as Gödel's three-valued logic [11]), the first component of an SE-interpretation is identified with the world "here", whilst the second component refers to the world "there". We write $S E(P)$ to refer to the set of all SE-models of a program $P$.

A semantical characterisation for hyperequivalence, similar to that using SE-models for strong equivalence, is given by the concept of an $H E$-model [5]. ${ }^{1}$ For $\mathcal{H}, \mathcal{B}, X, Y \subseteq$ $A t$, an SE-interpretation $(X, Y)$ is an $H E$-model relative to $\langle\mathcal{H}, \mathcal{B}\rangle$ of a DLP $P$ over $A t$, or an $\langle\mathcal{H}, \mathcal{B}\rangle$-model of $P$ for short, iff (i) $Y=P$, (ii) for all $Y^{\prime} \subset Y$ with $Y^{\prime} \cap \mathcal{H}=Y \cap$ $\mathcal{H}$, it holds that $Y^{\prime} \not \models P^{Y}$, (iii) if $X \subset Y$, there is an $X^{\prime} \subset Y$ with $X^{\prime} \cap(\mathcal{H} \cup \mathcal{B})=X$ such that $X^{\prime} \models P^{Y}$, and (iv) if $X \subset Y$, for each $X^{\prime} \subset Y$ with $(X \cap \mathcal{H}) \subseteq\left(X^{\prime} \cap \mathcal{H}\right)$, $\left(X^{\prime} \cap \mathcal{B}\right) \subseteq(X \cap \mathcal{B})$, and $X \neq X^{\prime} \cap(\mathcal{H} \cup \mathcal{B})$, it holds that $X^{\prime} \not P^{Y}$. The set of all $\langle\mathcal{H}, \mathcal{B}\rangle$-models of a program $P$ is denoted by $H E_{\mathcal{B}}^{\mathcal{H}}(P)$. Note that, for every non-total $\langle\mathcal{H}, \mathcal{B}\rangle$-model $(X, Y)$ of $P$, it holds that $(X \cap \mathcal{H}) \subset(Y \cap \mathcal{H})$ and $X \subset Y \cap(\mathcal{H} \cup \mathcal{B})$.

\footnotetext{
${ }^{1}$ We slightly rephrase the original definition of an HE-model.
} 
Proposition 1 ([5]). For all programs $P, Q$ over At and every $\mathcal{H}, \mathcal{B} \subseteq A t, P \equiv_{\mathcal{B}}^{\mathcal{H}} Q$ iff $H E_{\mathcal{B}}^{\mathcal{H}}(P)=H E_{\mathcal{B}}^{\mathcal{H}}(Q)$.

\section{Setting the Stage: Casting under Strong Equivalence}

The main question addressed in this paper is expressed by the following parametrised decision problem:

Definition 1. Let $\mathcal{C}$ be a class of programs over some set At of atoms. Then, $\mathrm{CAST}(\mathcal{C})$ is the problem of deciding whether, for a given $D L P P$ over At and sets $\mathcal{H}, \mathcal{B} \subseteq$ At of atoms, there exists a program $Q \in \mathcal{C}$ with $P \equiv_{\mathcal{B}}^{\mathcal{H}} Q$.

If a program $P$ together with sets $\mathcal{H}, \mathcal{B}$ of atoms constitute a yes-instance of $\operatorname{CAST}(\mathcal{C})$, we say that $P$ is castable to $\mathcal{C}$ under $\langle\mathcal{H}, \mathcal{B}\rangle$-equivalence. Furthermore, a program $Q \in$ $\mathcal{C}$ with $P \equiv_{\mathcal{B}}^{\mathcal{H}} Q$ is called an $\langle\mathcal{H}, \mathcal{B}\rangle$-casting of $P$ to $\mathcal{C}$. In this paper, we are interested in those versions of $\operatorname{CAST}(\mathcal{C})$ where $\mathcal{C} \in\{\mathcal{N} \mathcal{L} \mathcal{P}, \mathcal{P} \mathcal{L} \mathcal{P}, \mathcal{H O} \mathcal{R} \mathcal{N}\}$. Our aim is to find model-theoretic conditions precisely characterising the yes-instances of $\operatorname{CAST}(\mathcal{C})$. We do so by providing, for each class $\mathcal{C}$ as above and sets $\mathcal{H}, \mathcal{B}$ of atoms, a property $\phi_{\mathcal{B}, \mathcal{C}}^{\mathcal{H}}(\cdot)$ satisfying the following key condition:

$(\star) \phi_{\mathcal{B}, \mathcal{C}}^{\mathcal{H}}(\mathcal{S})$ holds iff there exists a program $Q \in \mathcal{C}$ with $\mathcal{S}=H E_{\mathcal{B}}^{\mathcal{H}}(Q)$, for each set $\mathcal{S}$ of SE-interpretations.

From this, we immediately get that, for a given DLP $P, \phi_{\mathcal{B}, \mathcal{C}}^{\mathcal{H}}\left(H E_{\mathcal{B}}^{\mathcal{H}}(P)\right)$ holds iff there exists a program $Q \in \mathcal{C}$ such that $H E_{\mathcal{B}}^{\mathcal{H}}(P)=H E_{\mathcal{B}}^{\mathcal{H}}(Q)$, which in turn implies that $\phi_{\mathcal{B}, \mathcal{C}}^{\mathcal{H}}\left(H E_{\mathcal{B}}^{\mathcal{H}}(P)\right)$ holds iff $P$ together with $\mathcal{H}$ and $\mathcal{B}$ is a yes-instance of CAST $(\mathcal{C})$, representing our desired characterisation. In addition to deciding whether an $\langle\mathcal{H}, \mathcal{B}\rangle$-casting of $P$ exists, we also provide constructive methods to obtain such castings.

As a preparatory step towards the general setting, in this section we deal with the case where $\mathcal{H}=\mathcal{B}=A t$, corresponding to casting under strong equivalence, which was already studied in the literature $[12,8,7]$. In particular, we will provide a special case of Condition $(\star)$, in terms of a property $\varphi_{\mathcal{C}}(\cdot)$, amounting to $\phi_{A t, \mathcal{C}}^{A t}(\cdot)$, as follows:

$\left(\star_{S E}\right) \varphi_{\mathcal{C}}(\mathcal{S})$ holds iff there exists a program $Q \in \mathcal{C}$ with $S E(Q)=\mathcal{S}$, for each set $\mathcal{S}$ of SE-interpretations.

We start with the following concept: A set $\mathcal{S}$ of SE-interpretations is well-defined iff, for each $(X, Y) \in \mathcal{S}$, also $(Y, Y) \in \mathcal{S}$. A well-defined set $\mathcal{S}$ of SE-interpretations is complete iff, for each $(X, Y) \in \mathcal{S}$, also $(X, Z) \in \mathcal{S}$, for any $Y \subseteq Z$ with $(Z, Z) \in \mathcal{S}$.

Proposition 2 ([12]). For each DLP $P, S E(P)$ is complete. Moreover, for any complete set $\mathcal{S}$ of $S E$-interpretations (over $A t$ ), there is a DLP $Q$ (over At) such that $S E(Q)=\mathcal{S}$.

Eiter, Tompits, and Woltran [12] describe, for a given complete set $\mathcal{S}$ of SE-interpretations, a concrete way for obtaining a DLP, $\mathrm{CP}_{\mathcal{S}}$, such that $S E\left(\mathrm{CP}_{\mathcal{S}}\right)=\mathcal{S}$ holds, which we refer to as the canonical program for $\mathcal{S}$. In fact, $\mathrm{CP}_{\mathcal{S}}$ is composed of rules $\leftarrow Y, \operatorname{not}(A t \backslash Y)$, for each $Y \subseteq A t$ such that $(Y, Y) \notin \mathcal{S}$, and of rules $\bigvee_{p \in(Y \backslash X)} p \leftarrow$ $X, \operatorname{not}(A t \backslash Y)$, for each $X \subset Y$ such that $(X, Y) \notin \mathcal{S}$ and $(Y, Y) \in \mathcal{S}$. 
To characterise programs castable to $\mathcal{N} \mathcal{L} \mathcal{P}$, we need an additional criterion on SEinterpretations: A set $\mathcal{S}$ of SE-interpretations is closed under here-intersection, or $\mathrm{HI}$ closed, iff, whenever $(X, Y) \in \mathcal{S}$ and $\left(X^{\prime}, Y\right) \in \mathcal{S}$, then $\left(X \cap X^{\prime}, Y\right) \in \mathcal{S}$. This property results from the facts that the reduct of a program relative to a set of atoms is a Horn program if disjunction is not involved, and that the models of Horn theories are closed under intersection.

The characterising property for DLPs that can be casted to $\mathcal{P} \mathcal{L} \mathcal{P}$ under strong equivalence is called here-totality: A set $\mathcal{S}$ of SE-interpretations is here-total iff, for any pair $(X, Y) \in \mathcal{S}$, it holds that $(X, X) \in \mathcal{S}$.

In order for a DLP $P$ to be castable to $\mathcal{H O R N}$ under strong equivalence, it is not sufficient that $S E(P)$ is both HI-closed and here-total. In fact, the transformations to an NLP introduce in general negations, and those to a PLP disjunctions. Additionally, it turns out that the property of closure under there-intersection is required: A set $\mathcal{S}$ of SEinterpretations is closed under there-intersection, or TI-closed, iff, whenever $(X, X) \in$ $\mathcal{S}$ and $(Y, Y) \in \mathcal{S}$, then $(X \cap Y, X \cap Y) \in \mathcal{S}$. Note that a set of SE-interpretations which is here-total and TI-closed is also HI-closed.

The next proposition states how the defined properties characterise castable DLPs.

Proposition $3([8,7])$. Let $P$ be a DLP over set of atoms At. Then, there exists $(i)$ an $N L P Q$ with $P \equiv{ }_{A t}^{A t} Q$ iff $S E(P)$ is HI-closed, (ii) a PLP $Q^{\prime}$ with $P \equiv_{A t}^{A t} Q^{\prime}$ iff $S E(P)$ is here-total, and (iii) a Horn program $Q^{\prime \prime}$ with $P \equiv_{A t}^{A t} Q^{\prime \prime}$ iff $S E(P)$ is here-total and TI-closed.

Example 1. Consider programs $P_{1}=\{b \vee c \leftarrow a ; a \leftarrow$ not $b ; b \leftarrow$ not $a ; b \leftarrow$ not $c\}$ and $P_{2}=\{a \vee b \leftarrow c ; b \leftarrow a ; a \leftarrow b$, not $c ; c \leftarrow b, \operatorname{not} a ; \leftarrow a, b, c\}$ over $\{a, b, c\}$. The sets of SE-models of these programs are ${ }^{2}$

$$
\begin{aligned}
& S E\left(P_{1}\right)=\{(b, b),(b, a b),(a b, a b),(a c, a c),(b, b c),(b c, b c),(\emptyset, a b c),(b, a b c),(c, a b c),(a b, a b c),(a c, a b c),(b c, a b c),(a b c, a b c)\} \text { and } \\
& S E\left(P_{2}\right)=\{(\emptyset, \emptyset),(\emptyset, a b),(a b, a b),(\emptyset, b c),(b c, b c)\} .
\end{aligned}
$$

$S E\left(P_{1}\right)$ is not HI-closed as it contains $(a b, a b c),(a c, a b c)$, but not $(a, a b c)$. Hence, for each NLP $Q, P_{1} \not F_{A t}^{A t} Q . S E\left(P_{2}\right)$ is HI-closed and $P_{2} \equiv{ }_{A t}^{A t} P_{2}^{\prime}$ holds for NLP $P_{2}^{\prime}=\{a \leftarrow b$, not $c ; c \leftarrow b$, not $a ; b \leftarrow a ; b \leftarrow c ; \leftarrow a, b, c\}$.

For creating a strongly equivalent NLP for a given DLP $P$ with $S E(P)$ being HI-closed, we refer to a known technique for removing disjunctions under strong equivalence [8].

Example 2. For program $P_{1}$ from Example 1, $S E\left(P_{1}\right)$ is not here-total, since, e.g., $(\emptyset, a b c) \in S E\left(P_{1}\right)$ but $(\emptyset, \emptyset) \notin S E\left(P_{1}\right)$. Hence, there is no strongly equivalent PLP. Consider program $P_{3}=\{a \leftarrow$ not $b ; b \leftarrow$ not $a ; \leftarrow a, b\}$ over $\{a, b\}$ with $S E\left(P_{3}\right)=$ $\{(a, a),(b, b)\}$ that is here-total. $P_{3}$ can be replaced by the strongly equivalent PLP $P_{3}^{\prime}=\{a \vee b \leftarrow ; \leftarrow a, b\}$.

Note that $P_{3}^{\prime}$ is obtained from $P_{3}$ by moving all atoms from $B^{-}(r)$ of each rule $r$ to $H(r)$. This transformation is called the left-shift of a program. Generally, the leftshift of any DLP $P$, where $S E(P)$ is here-total, is strongly equivalent to $P$.

\footnotetext{
${ }^{2}$ We write " $a b c$ " instead of " $\{a, b, c\}$ ", " $a$ " instead of " $\{a\}$ ", etc.
} 
Example 3. For program $P_{2}$ from Example 1, $S E\left(P_{2}\right)$ is HI-closed, here-total, but not TI-closed, as $(a b, a b),(b c, b c) \in S E\left(P_{2}\right)$ but $(b, b) \notin S E\left(P_{2}\right)$. Hence, there is no Horn program which is strongly equivalent to $P_{2}$. Consider program $P_{4}=\{a \vee b \leftarrow ; a \leftarrow$ $b$, not $c ; \leftarrow b, c\}$ over $\{a, b, c\}$, where $S E\left(P_{4}\right)=\{(a, a),(a, a b),(a b, a b),(a, a c)$, $(a c, a c)\}$ is both here-total and TI-closed. $P_{4}$ can be replaced by the strongly equivalent Horn program $P_{4}^{\prime}=\{a \leftarrow ; \leftarrow b, c\}$.

To obtain a Horn program $Q$ such that $P \equiv_{\mathcal{B}}^{\mathcal{H}} Q$ for a given DLP $P$, we proceed in two steps. First, a left-shift is applied on $P$ to obtain a strongly equivalent PLP. Then, the remaining disjunctions can be eliminated by removing atoms from rule heads, as suggested by the following theorem.

Theorem 1. For every PLP P such that $S E(P)$ is TI-closed, a Horn program $P^{\prime}$ such that $P{ }_{A t}^{A t} P^{\prime}$ can be obtained by removing all but one atom, a, from the head of each rule $r \in P$ with $|H(r)|>1$. Thereby, a has to be chosen in such a way that, for every model $Y$ of $P$ with $B(r) \subseteq Y, a \in Y$ holds.

For lifting the results for strong equivalence to general hyperequivalence, we will need the following consequence of Propositions 2 and 3.

Theorem 2. Let $\mathcal{S}$ be a set of SE-interpretations. Then, there exists

- an NLP $Q$ with $S E(Q)=\mathcal{S}$ iff $\mathcal{S}$ is complete and HI-closed,

- a PLP $Q^{\prime}$ with $S E\left(Q^{\prime}\right)=\mathcal{S}$ iff $\mathcal{S}$ is complete and here-total, and

- a Horn program $Q^{\prime \prime}$ with $S E\left(Q^{\prime \prime}\right)=\mathcal{S}$ iff $\mathcal{S}$ is complete, here-total, and TI-closed.

\section{Main Results}

We now lift the results for strong equivalence to hyperequivalence. Note that Theorem 2 provides us with the special case $\left(\star_{S E}\right)$ of our key condition $(\star)$, expressing that a property $\varphi_{\mathcal{C}}(\mathcal{S})$ holds iff there exists a program $Q \in \mathcal{C}$ with $S E(Q)=\mathcal{S}$, for each $\mathcal{C} \in\{\mathcal{N} \mathcal{L} \mathcal{P}, \mathcal{P} \mathcal{L} \mathcal{P}, \mathcal{H} \mathcal{O} \mathcal{R} \mathcal{N}\}$. We will use this property for proving the general case of Condition $(\star)$, thus establishing our main results, as outlined in the beginning of Section 3. This is achieved as follows: First of all, for each $\mathcal{C}$ as above, we define the corresponding property $\phi_{\mathcal{B}, \mathcal{C}}^{\mathcal{H}}(\cdot)$ together with a function $\tau_{\mathcal{B}, \mathcal{C}}^{\mathcal{H}}(\cdot)$ from sets of SEinterpretations to sets of SE-interpretations, which we refer to as a completion transformation, such that the following properties hold:

(i) if, for a set $\mathcal{S}$ of SE-interpretations, $\phi_{\mathcal{B}, \mathcal{C}}^{\mathcal{H}}(\mathcal{S})$ holds, then $\varphi_{\mathcal{C}}\left(\tau_{\mathcal{B}, \mathcal{C}}^{\mathcal{H}}(\mathcal{S})\right)$ holds;

(ii) if, for a set $\mathcal{S}$ of SE-interpretations, $\phi_{\mathcal{B}, \mathcal{C}}^{\mathcal{H}}(\mathcal{S})$ holds and there exists a program $Q \in \mathcal{C}$ such that $\tau_{\mathcal{B}, \mathcal{C}}^{\mathcal{H}}(\mathcal{S})=S E(Q)$, then $H E_{\mathcal{B}}^{\mathcal{H}}(Q)=\mathcal{S}$

(iii) for every program $Q \in \mathcal{C}, \phi_{\mathcal{B}, \mathcal{C}}^{\mathcal{H}}\left(H E_{\mathcal{B}}^{\mathcal{H}}(Q)\right)$ holds.

From these properties, $(\star)$ can then be established as follows: Assume that $\phi_{\mathcal{B}, \mathcal{C}}^{\mathcal{H}}(\mathcal{S})$ holds. Then, by (i), so does $\varphi_{\mathcal{C}}\left(\tau_{\mathcal{B}, \mathcal{C}}^{\mathcal{H}}(\mathcal{S})\right)$. From property $\left(\star_{S E}\right)$ it follows that there is a program $Q \in \mathcal{C}$ with $S E(Q)=\tau_{\mathcal{B}, \mathcal{C}}^{\mathcal{H}}(\mathcal{S})$. Hence, by (ii), $H E_{\mathcal{B}}^{\mathcal{H}}(Q)=\mathcal{S}$. Conversely, let $Q \in \mathcal{C}$ be a program with $H E_{\mathcal{B}}^{\mathcal{H}}(Q)=\mathcal{S}$. By (iii), $\phi_{\mathcal{B}, \mathcal{C}}^{\mathcal{H}}\left(H E_{\mathcal{B}}^{\mathcal{H}}(Q)\right)$ holds. Since 
$H E_{\mathcal{B}}^{\mathcal{H}}(Q)=\mathcal{S}$, we get that $\phi_{\mathcal{B}, \mathcal{C}}^{\mathcal{H}}(\mathcal{S})$ holds. Hence, $(\star)$ holds. As argued in Section 3, $(\star)$ in turn implies our main result expressing that, for each $\mathcal{C} \in\{\mathcal{N} \mathcal{L P}, \mathcal{P} \mathcal{L P}, \mathcal{H O R} \mathcal{N}\}$, $\phi_{\mathcal{B}, \mathcal{C}}^{\mathcal{H}}\left(H E_{\mathcal{B}}^{\mathcal{H}}(P)\right)$ holds iff $P, \mathcal{H}$, and $\mathcal{B}$ constitute a yes-instance of $\operatorname{CAST}(\mathcal{C})$.

In the remainder of this section we will show, for each individual class $\mathcal{C}$, how $\phi_{\mathcal{B}, \mathcal{C}}^{\mathcal{H}}(\cdot)$ and $\tau_{\mathcal{B}, \mathcal{C}}^{\mathcal{H}}(\cdot)$ are defined. We will provide the main proofs for the case of casting to $\mathcal{N} \mathcal{L P}$ but only show the constructions and formulate the main results for the cases of casting to $\mathcal{P} \mathcal{L P}$ and $\mathcal{H O R N}$ due to space limitations.

\subsection{Completeness for Hyperequivalence Models}

First, we will introduce the notion of $\langle\mathcal{H}, \mathcal{B}\rangle$-completeness, a property of sets of SEinterpretations which is characteristic for the set of all $\langle\mathcal{H}, \mathcal{B}\rangle$-models of a program.

Definition 2. Let $\mathcal{H}, \mathcal{B}$ be sets of atoms and $\mathcal{S}$ a set of SE-interpretations. Then, $\mathcal{S}$ is $\langle\mathcal{H}, \mathcal{B}\rangle$-well-defined if, for each $(X, Y) \in \mathcal{S}$ such that $X \subset Y$, it holds that $(i)(Y, Y) \in$ $\mathcal{S}$, (ii) $X \subset(Y \cap(\mathcal{H} \cup \mathcal{B}))$, (iii) $(X \cap \mathcal{H}) \subset(Y \cap \mathcal{H})$, and (iv) there is no $\left(X^{\prime}, Y\right) \in \mathcal{S}$ with $X^{\prime} \subset Y,(X \cap \mathcal{H}) \subseteq\left(X^{\prime} \cap \mathcal{H}\right),\left(X^{\prime} \cap \mathcal{B}\right) \subseteq(X \cap \mathcal{B})$, and $X \neq X^{\prime}$.

Moreover, $\mathcal{S}$ is $\langle\mathcal{H}, \mathcal{B}\rangle$-complete if $\mathcal{S}$ is $\langle\mathcal{H}, \mathcal{B}\rangle$-well-defined and, for all $X, Y, Z$ such that $(X, Y),(Z, Z) \in \mathcal{S}$ and $Y \subset Z$, there is some $X^{\prime} \subset Z$ such that $\left(X^{\prime}, Z\right) \in \mathcal{S}$, $(X \cap \mathcal{H}) \subseteq\left(X^{\prime} \cap \mathcal{H}\right)$, and $\left(X^{\prime} \cap \mathcal{B}\right) \subseteq(X \cap \mathcal{B})$.

Condition (i) of $\langle\mathcal{H}, \mathcal{B}\rangle$-well-definedness subsumes well-definedness. Conditions (ii) and (iii) reflect that the "there"-component $Y$ of an $\langle\mathcal{H}, \mathcal{B}\rangle$-model of $P$ is a model of $P^{Y}$, minimal amongst the models sharing the same atoms from $\mathcal{H}$, and express that the "here"-component of non-total $\langle\mathcal{H}, \mathcal{B}\rangle$-models is a subset of $\mathcal{H} \cup \mathcal{B}$. Observe that Condition (iv) expresses an optimality property of non-total $\langle\mathcal{H}, \mathcal{B}\rangle$-models with respect to sets $\mathcal{H}$ and $\mathcal{B}$. For relating $\langle\mathcal{H}, \mathcal{B}\rangle$-models to SE-models, this optimality criterion is also captured in the following notion:

Definition 3. Let $\mathcal{H}, \mathcal{B}$ be sets of atoms and $\mathcal{S}$ a set of $S E$-interpretations. Then, a nontotal SE-interpretation $(X, Y) \in \mathcal{S}$ is $\langle\mathcal{H}, \mathcal{B}\rangle$-optimal in $\mathcal{S}$ if there is no $X^{\prime} \subset Y$ such that $\left(X^{\prime}, Y\right) \in \mathcal{S},(X \cap \mathcal{H}) \subseteq\left(X^{\prime} \cap \mathcal{H}\right),\left(X^{\prime} \cap \mathcal{B}\right) \subseteq(X \cap \mathcal{B})$, and $X \cap(\mathcal{H} \cup \mathcal{B}) \neq$ $X^{\prime} \cap(\mathcal{H} \cup \mathcal{B})$.

The following two lemmas express relations between SE-models and HE-models.

Lemma 1. Let $\mathcal{H}, \mathcal{B} \subseteq$ At be sets of atoms, $P$ a DLP over At, and $(X, Y)$ a nontotal SE-model of $P$ that is $\langle\mathcal{H}, \mathcal{B}\rangle$-optimal in $S E(P)$. If $(Y, Y) \in H E_{\mathcal{B}}^{\mathcal{H}}(P)$, then $(X \cap(\mathcal{H} \cup \mathcal{B}), Y) \in H E_{\mathcal{B}}^{\mathcal{H}}(P)$.

Lemma 2. Let $\mathcal{H}, \mathcal{B} \subseteq$ At be sets of atoms and $P$ a DLP over At. If $(Y, Y)$ is an $\langle\mathcal{H}, \mathcal{B}\rangle$-model of $P$, then $(Y, Y)$ is an SE-model of $P$. If $(X, Y)$ is a non-total $\langle\mathcal{H}, \mathcal{B}\rangle$ model of $P$, then, for some $X^{\prime} \subset Y$ with $X=X^{\prime} \cap(\mathcal{H} \cup \mathcal{B}),\left(X^{\prime}, Y\right) \in S E(P)$ and $\left(X^{\prime}, Y\right)$ is $\langle\mathcal{H}, \mathcal{B}\rangle$-optimal in $S E(P)$.

With Lemmas 1 and 2 at hand, we can show the following result for DLPs.

Theorem 3. Let $\mathcal{H}, \mathcal{B} \subseteq$ At be sets of atoms and $P$ a DLP over At. Then, $H E_{\mathcal{B}}^{\mathcal{H}}(P)$ is $\langle\mathcal{H}, \mathcal{B}\rangle$-complete. 
Proof. First, we show that $H E_{\mathcal{B}}^{\mathcal{H}}(P)$ is $\langle\mathcal{H}, \mathcal{B}\rangle$-well-defined. Consider some $X \subset Y$ with $(X, Y) \in H E_{\mathcal{B}}^{\mathcal{H}}(P)$. Conditions (i) and (ii) of the definition of an $\langle\mathcal{H}, \mathcal{B}\rangle$-model are satisfied for $(Y, Y)$. As $(Y, Y)$ is total, also Conditions (iii) and (iv) hold, and consequently $(Y, Y) \in H E_{\mathcal{B}}^{\mathcal{H}}(P)$. Since $(X, Y)$ is non-total, we have that $(X \cap \mathcal{H}) \subset$ $(Y \cap \mathcal{H})$ and $X \subset Y \cap(\mathcal{H} \cup \mathcal{B})$. Towards a contradiction, assume that there is some $(U, Y) \in H E_{\mathcal{B}}^{\mathcal{H}}(P)$ with $U \subset Y,(X \cap \mathcal{H}) \subseteq(U \cap \mathcal{H}),(U \cap \mathcal{B}) \subseteq(X \cap \mathcal{B})$, and $X \neq U$. From $(U, Y) \in H E_{\mathcal{B}}^{\mathcal{H}}(P)$ it follows from Condition (iii) of the definition of an $\langle\mathcal{H}, \mathcal{B}\rangle$-model of $P$ that there is some $U^{\prime} \subset Y$ with $U^{\prime} \models P^{Y}$ and $U^{\prime} \cap(\mathcal{H} \cap \mathcal{B})=U$. From the latter and $X \neq U$, we get $X \neq U^{\prime} \cap(\mathcal{H} \cap \mathcal{B})$. Furthermore, it holds that $(X \cap \mathcal{H}) \subseteq\left(U^{\prime} \cap \mathcal{H}\right)$ and $\left(U^{\prime} \cap \mathcal{B}\right) \subseteq(X \cap \mathcal{B})$. Since $(X, Y) \in H E_{\mathcal{B}}^{\mathcal{H}}(P)$, we get by Condition (iv) that $U^{\prime} \not P^{Y}$, being a contradiction to our previous result that $U^{\prime} \models P^{Y}$. Hence, $H E_{\mathcal{B}}^{\mathcal{H}}(P)$ is $\langle\mathcal{H}, \mathcal{B}\rangle$-well-defined. Towards a contradiction, assume there is a DLP $P$ such that $H E_{\mathcal{B}}^{\mathcal{H}}(P)$ is not $\langle\mathcal{H}, \mathcal{B}\rangle$-complete. Observe that there must be some $X, Y, Z$ with $Y \subset Z$ such that $(X, Y),(Z, Z) \in H E_{\mathcal{B}}^{\mathcal{H}}(P)$ and, for every $X^{\prime} \subset Z$ with $(X \cap \mathcal{H}) \subseteq\left(X^{\prime} \cap \mathcal{H}\right)$ and $\left(X^{\prime} \cap \mathcal{B}\right) \subseteq(X \cap \mathcal{B})$, it holds that $\left(X^{\prime}, Z\right) \notin H E_{\mathcal{B}}^{\mathcal{H}}(P)$. Now we show that there is some $X^{\prime} \subset Z$ such that $X^{\prime} \cap(\mathcal{H} \cup \mathcal{B})=X \cap(\mathcal{H} \cup \mathcal{B})$ and $\left(X^{\prime}, Z\right) \in S E(P)$. Consider the case that $X=Y$. We know from Lemma 2 that $(X, X)$ and $(Z, Z)$ are SE-models of $P$. By completeness of SE-models, we get that $(X, Z) \in S E(P)$. Note that $X \subset Z$. Now assume that $X \subset Y$. Then, by Lemma 2, there is a $U \subset Z$ with $X=U \cap(\mathcal{H} \cup \mathcal{B})$ such that $(U, Y) \in S E(P)$. Thus, since $(Z, Z) \in S E(P)$ and it holds that $Y \subseteq Z$, we get by completeness of SE-models that $(U, Z) \in S E(P)$. Consequently, in either case, there exists some $X^{\prime} \subset Z$ such that $X^{\prime} \cap(\mathcal{H} \cup \mathcal{B})=X \cap(\mathcal{H} \cup \mathcal{B})$ and $\left(X^{\prime}, Z\right) \in S E(P)$. From that, and by definition of $\langle\mathcal{H}, \mathcal{B}\rangle$-optimality, there exists an $\langle\mathcal{H}, \mathcal{B}\rangle$-optimal $X_{\text {opt }} \subset Z$ in $S E(P)$ with $(X \cap \mathcal{H}) \subseteq$ $\left(X_{\text {opt }} \cap \mathcal{H}\right)$ and $\left(X_{\text {opt }} \cap \mathcal{B}\right) \subseteq(X \cap \mathcal{B})$. By $\langle\mathcal{H}, \mathcal{B}\rangle$-optimality of $X_{\text {opt }}$ in $S E(P)$, since $(Z, Z) \in H E_{\mathcal{B}}^{\mathcal{H}}(P)$, we get by Lemma 1 that $\left(X_{\text {opt }} \cap(\mathcal{H} \cup \mathcal{B}), Z\right) \in H E_{\mathcal{B}}^{\mathcal{H}}(P)$. However, as $(X \cap \mathcal{H}) \subseteq\left(X_{\text {opt }} \cap \mathcal{H}\right)$ and $\left(X_{\text {opt }} \cap \mathcal{B}\right) \subseteq(X \cap \mathcal{B})$, this is a contradiction to our observation. Hence, $H E_{\mathcal{B}}^{\mathcal{H}}(P)$ is $\langle\mathcal{H}, \mathcal{B}\rangle$-complete.

Next, we show that, conversely, for every $\langle\mathcal{H}, \mathcal{B}\rangle$-complete set $\mathcal{S}$ of SE-interpretations, there is a DLP $P$ such that $H E_{\mathcal{B}}^{\mathcal{H}}(P)=\mathcal{S}$. To this end, we define a mapping $\mathrm{c}_{\mathcal{H}, \mathcal{B}}(\cdot)$, representing the completion transformation $\tau_{\mathcal{B}, \mathcal{C}}^{\mathcal{H}}(\cdot)$ for the case $\mathcal{C}=\mathcal{N} \mathcal{L} \mathcal{P}$ mentioned at the beginning of this section, assigning sets of SE-interpretations to sets of SE-interpretations, serving a double role: On the one hand, it is a device to construct a DLP from any $\langle\mathcal{H}, \mathcal{B}\rangle$-complete set $\mathcal{S}$ of SE-interpretations. On the other hand, the rewriting is designed such that $\mathrm{c}_{\mathcal{H}, \mathcal{B}}(\mathcal{S})$ is HI-closed iff $\mathcal{S}$ is $\langle\mathcal{H}, \mathcal{B}\rangle$-closed under hereintersection, a property that is described later in this section.

Definition 4. Let $\mathcal{H}, \mathcal{B}$ be sets of atoms. For every set $\mathcal{S}$ of SE-interpretations, let $\mathrm{c}_{\mathcal{H}, \mathcal{B}}(\mathcal{S})$ be given by $\{(Y, Y) \mid(Y, Y) \in \mathcal{S}\} \cup\{(X, Y) \mid X \subset Y,(X \cap \mathcal{H}) \subseteq$ $\left.\left(X^{\prime} \cap \mathcal{H}\right),\left(X^{\prime} \cap \mathcal{B}\right) \subseteq(X \cap \mathcal{B}),\left(X^{\prime}, Y\right) \in \mathcal{S}, X^{\prime} \subset Y\right\}$.

Note that whenever $(X, Y) \in \mathcal{S}$, also $(X, Y) \in \mathrm{c}_{\mathcal{H}, \mathcal{B}}(\mathcal{S})$.

Lemma 3. Let $\mathcal{H}, \mathcal{B}$ be sets of atoms and $\mathcal{S}$ a set of $S E$-interpretations. If $\mathcal{S}$ is $\langle\mathcal{H}, \mathcal{B}\rangle$ complete, then $\mathrm{c}_{\mathcal{H}, \mathcal{B}}(\mathcal{S})$ is complete. 
Proof. Assume that $\mathcal{S}$ is $\langle\mathcal{H}, \mathcal{B}\rangle$-complete. First, we show that $\mathrm{c}_{\mathcal{H}, \mathcal{B}}(\mathcal{S})$ is well-defined. Consider some $(X, Y) \in \mathrm{c}_{\mathcal{H}, \mathcal{B}}(\mathcal{S})$ with $X \subset Y$. By Definition 4, there is some $\left(X^{\prime}, Y\right) \in \mathcal{S}$. Since $\mathcal{S}$ is well-defined, we also have $(Y, Y) \in \mathcal{S}$ and therefore $(Y, Y) \in$ $\mathrm{c}_{\mathcal{H}, \mathcal{B}}(\mathcal{S})$. So, $\mathrm{c}_{\mathcal{H}, \mathcal{B}}(\mathcal{S})$ is well-defined. Towards a contradiction, assume that $\mathrm{c}_{\mathcal{H}, \mathcal{B}}(\mathcal{S})$ is not complete. There must be some interpretations $X, Y, Z$ with $Y \subseteq Z$ such that $(X, Y),(Z, Z) \in \mathrm{c}_{\mathcal{H}, \mathcal{B}}(\mathcal{S})$ and $(X, Z) \notin \mathrm{c}_{\mathcal{H}, \mathcal{B}}(\mathcal{S})$. Note that by Definition 4, it holds that $(Z, Z) \in \mathcal{S}$. From $(X, Y) \in \mathrm{c}_{\mathcal{H}, \mathcal{B}}(\mathcal{S})$, it follows that $X \subseteq Y$. Furthermore, it must hold that $Y \subset Z$, as otherwise $(X, Z)=(X, Y) \in \mathrm{c}_{\mathcal{H}, \mathcal{B}}(\mathcal{S})$. In case that $X=Y$, we get by Definition 4 that $(X, X) \in \mathcal{S}$. Since $(Z, Z) \in \mathcal{S}$, by $\langle\mathcal{H}, \mathcal{B}\rangle$-completeness of $\mathcal{S}$ there is an $X^{\prime} \subset Z$, where $\left(X^{\prime}, Z\right) \in \mathcal{S},(X \cap \mathcal{H}) \subseteq\left(X^{\prime} \cap \mathcal{H}\right)$ and $\left(X^{\prime} \cap \mathcal{B}\right) \subseteq(X \cap \mathcal{B})$. In case that $X \subset Y$, we get by Definition 4 that there is some $\left(X^{\prime \prime}, Y\right) \in \mathcal{S}$ such that $(X \cap \mathcal{H}) \subseteq\left(X^{\prime \prime} \cap \mathcal{H}\right)$ and $\left(X^{\prime \prime} \cap \mathcal{B}\right) \subseteq(X \cap \mathcal{B})$. Since $(Z, Z) \in \mathcal{S}$, by $\langle\mathcal{H}, \mathcal{B}\rangle$ completeness of $\mathcal{S}$, there is again an $X^{\prime} \subset Z$ with $\left(X^{\prime}, Z\right) \in \mathcal{S}$, where $(X \cap \mathcal{H}) \subseteq$ $\left(X^{\prime \prime} \cap \mathcal{H}\right) \subseteq\left(X^{\prime} \cap \mathcal{H}\right)$ and $\left(X^{\prime} \cap \mathcal{B}\right) \subseteq\left(X^{\prime \prime} \cap \mathcal{B}\right) \subseteq(X \cap \mathcal{B})$. Therefore, in both cases, we have $(X, Z) \in \mathrm{c}_{\mathcal{H}, \mathcal{B}}(\mathcal{S})$, being a contradiction to $(X, Z) \notin \mathrm{c}_{\mathcal{H}, \mathcal{B}}(\mathcal{S})$. Hence, $\mathrm{c}_{\mathcal{H}, \mathcal{B}}(\mathcal{S})$ is complete.

The next lemma shows that a program having $\mathrm{c}_{\mathcal{H}, \mathcal{B}}(\mathcal{S})$ as its set of SE-models is guaranteed to have $\mathcal{S}$ as its set of $\langle\mathcal{H}, \mathcal{B}\rangle$-models. This is the case since all freshly introduced SE-interpretations either lack $\langle\mathcal{H}, \mathcal{B}\rangle$-optimality in $\mathrm{c}_{\mathcal{H}, \mathcal{B}}(\mathcal{S})$ or are already represented by an SE-interpretation in $\mathcal{S}$.

Lemma 4. Let $\mathcal{H}, \mathcal{B} \subseteq$ At be sets of atoms, $\mathcal{S}$ a set of SE-interpretations, and $P$ a DLP over At. If $S E(P)=\mathrm{c}_{\mathcal{H}, \mathcal{B}}(\mathcal{S})$ and $\mathcal{S}$ is $\langle\mathcal{H}, \mathcal{B}\rangle$-complete, then $H E_{\mathcal{B}}^{\mathcal{H}}(P)=\mathcal{S}$.

Now we put things together. By using Proposition 2 and Lemmas 3 and 4 we get the following result:

Theorem 4. Let $\mathcal{H}, \mathcal{B} \subseteq$ At be sets of atoms. Then, for every $\langle\mathcal{H}, \mathcal{B}\rangle$-complete set $\mathcal{S}$ of $S E$-interpretations, there is a DLP P over At such that $H_{\mathcal{B}}^{\mathcal{H}}(P)=\mathcal{S}$.

\subsection{Elimination of Disjunction}

While $\langle\mathcal{H}, \mathcal{B}\rangle$-completeness generally characterises sets of SE-interpretations that are $\langle\mathcal{H}, \mathcal{B}\rangle$-models of a DLP, we now define a supplementary property for NLPs.

Definition 5. Let $\mathcal{H}$ and $\mathcal{B}$ be sets of atoms. A set $\mathcal{S}$ of SE-interpretations is $\langle\mathcal{H}, \mathcal{B}\rangle$ closed under here-intersection, or $\langle\mathcal{H}, \mathcal{B}\rangle$-HI-closed, if, whenever $\left(X_{1}, Y\right) \in \mathcal{S}$ and $\left(X_{2}, Y\right) \in \mathcal{S}$, with $X_{1} \subset Y$ and $X_{2} \subset Y$, there is some $X^{\prime} \subset Y$ such that $\left(X^{\prime}, Y\right) \in \mathcal{S}$, $\left(X_{1} \cap X_{2}\right) \cap \mathcal{H} \subseteq\left(X^{\prime} \cap \mathcal{H}\right)$, and $\left(X^{\prime} \cap \mathcal{B}\right) \subseteq\left(X_{1} \cap X_{2}\right) \cap \mathcal{B}$.

In terms of the discussion from the beginning of this section, the joint stipulation of $\langle\mathcal{H}, \mathcal{B}\rangle$-completeness and $\langle\mathcal{H}, \mathcal{B}\rangle$-closure under here-intersection amounts to condition $\phi_{\mathcal{B}, \mathcal{C}}^{\mathcal{H}}(\cdot)$ for $\mathcal{C}=\mathcal{N} \mathcal{L} \mathcal{P}$.

Theorem 5. Let $\mathcal{H}, \mathcal{B} \subseteq$ At be sets of atoms and $P$ an NLP over At. Then, $H E_{\mathcal{B}}^{\mathcal{H}}(P)$ is $\langle\mathcal{H}, \mathcal{B}\rangle$-HI-closed. 
Proof. Towards a contradiction, assume $H E_{\mathcal{B}}^{\mathcal{H}}(P)$ is not $\langle\mathcal{H}, \mathcal{B}\rangle$-HI-closed. Then, there must be some $X_{1}, X_{2}, Y$ such that $\left(X_{1}, Y\right),\left(X_{2}, Y\right) \in H E_{\mathcal{B}}^{\mathcal{H}}(P)$ with $X_{1} \subset Y$ and $X_{2} \subset Y$, but there is no $X^{\prime} \subset Y$ such that $\left(X^{\prime}, Y\right) \in H E_{\mathcal{B}}^{\mathcal{H}}(P),\left(X_{1} \cap X_{2} \cap \mathcal{H}\right) \subseteq$ $\left(X^{\prime} \cap \mathcal{H}\right)$, and $\left(X^{\prime} \cap \mathcal{B}\right) \subseteq\left(X_{1} \cap X_{2} \cap \mathcal{B}\right)$. Note that therefore $\left(X_{1} \cap X_{2}, Y\right) \notin H E_{\mathcal{B}}^{\mathcal{H}}(P)$. Moreover, from well-definedness of $H E_{\mathcal{B}}^{\mathcal{H}}(P)$ and $\left(X^{\prime}, Y\right) \in H E_{\mathcal{B}}^{\mathcal{H}}(P)$, it follows that $(Y, Y) \in H E_{\mathcal{B}}^{\mathcal{H}}(P)$. By Lemma 2, there are SE-models $\left(X_{1}^{\prime}, Y\right),\left(X_{2}^{\prime}, Y\right)$ of $P$ such that $X_{1}^{\prime} \subset Y, X_{2}^{\prime} \subset Y, X_{1}=\left(X_{1}^{\prime} \cap(\mathcal{H} \cup \mathcal{B})\right)$, and $X_{2}=\left(X_{2}^{\prime} \cap(\mathcal{H} \cup \mathcal{B})\right)$. As $P$ is an NLP, from Theorem 2 it follows that $S E(P)$ is HI-closed. Therefore, $\left(X_{1}^{\prime} \cap X_{2}^{\prime}, Y\right) \in S E(P)$ holds. Note that Conditions (i) and (ii) of the definition for being an $\langle\mathcal{H}, \mathcal{B}\rangle$-model of $P$ are satisfied by $\left(X_{1} \cap X_{2}, Y\right)$ since $(Y, Y) \in H E_{\mathcal{B}}^{\mathcal{H}}(P)$. Furthermore, from $\left(X_{1}^{\prime} \cap X_{2}^{\prime}\right) \subset$ $Y,\left(X_{1}^{\prime} \cap X_{2}^{\prime} \cap(\mathcal{H} \cup \mathcal{B})\right)=\left(X_{1} \cap X_{2}\right)$, and $X_{1}^{\prime} \cap X_{2}^{\prime} \models P^{Y}$, it follows that Condition (iii) holds. Since $\left(X_{1} \cap X_{2}, Y\right) \notin H E_{\mathcal{B}}^{\mathcal{H}}(P)$, Condition (iv) must be violated. Hence, there is some $X^{\prime} \subset Y$ with $\left(X_{1} \cap X_{2} \cap \mathcal{H}\right) \subseteq\left(X^{\prime} \cap \mathcal{H}\right),\left(X^{\prime} \cap \mathcal{B}\right) \subseteq\left(X_{1} \cap X_{2} \cap \mathcal{B}\right)$, and $\left(X_{1} \cap X_{2}\right) \neq X^{\prime} \cap(\mathcal{H} \cup \mathcal{B})$ such that $X^{\prime} \models P^{Y}$, and thus $\left(X^{\prime}, Y\right) \in S E(P)$. Consider an $\langle\mathcal{H}, \mathcal{B}\rangle$-optimal SE-interpretation $\left(X_{\text {opt }}, Y\right)$ in $S E(P)$ having these properties. Then, since $(Z, Z) \in H E_{\mathcal{B}}^{\mathcal{H}}(P)$, we get by Lemma 1 that $\left(X_{\text {opt }} \cap(\mathcal{H} \cup \mathcal{B}), Y\right) \in H E_{\mathcal{B}}^{\mathcal{H}}(P)$. We end up in a contradiction, as $X_{\text {opt }} \cap(\mathcal{H} \cup \mathcal{B}) \subset Y,\left(X_{1} \cap X_{2} \cap \mathcal{H}\right) \subseteq\left(X_{\text {opt }} \cap \mathcal{H}\right)$, and $\left(X_{\text {opt }} \cap \mathcal{B}\right) \subseteq\left(X_{1} \cap X_{2} \cap \mathcal{B}\right)$. Hence, $H E_{\mathcal{B}}^{\mathcal{H}}(P)$ is $\langle\mathcal{H}, \mathcal{B}\rangle$-HI-closed.

As shown next, for an $\langle\mathcal{H}, \mathcal{B}\rangle$-complete set $\mathcal{S}$ of SE-interpretations that is $\langle\mathcal{H}, \mathcal{B}\rangle$ $\mathrm{HI}$-closed, $\mathrm{c}_{\mathcal{H}, \mathcal{B}}(\mathcal{S})$ is the set of SE-models of a normal logic program. New non-total SE-interpretations are introduced in $\mathrm{C}_{\mathcal{H}, \mathcal{B}}(\mathcal{S})$ that, as we will see below, guarantee that $\mathrm{C}_{\mathcal{H}, \mathcal{B}}(\mathcal{S})$ is HI-closed.

Lemma 5. Let $\mathcal{H}, \mathcal{B}$ be sets of atoms and $\mathcal{S}$ a set of $S E$-interpretations. If $\mathcal{S}$ is $\langle\mathcal{H}, \mathcal{B}\rangle$ HI-closed, then $\mathrm{C}_{\mathcal{H}, \mathcal{B}}(\mathcal{S})$ is HI-closed.

Proof. Let $\mathcal{S}$ be $\langle\mathcal{H}, \mathcal{B}\rangle$-HI-closed. Consider some $\left(X_{1}, Y\right),\left(X_{2}, Y\right) \in \mathrm{c}_{\mathcal{H}, \mathcal{B}}(\mathcal{S})$. We show that $\left(X_{1} \cap X_{2}, Y\right) \in \mathrm{c}_{\mathcal{H}, \mathcal{B}}(\mathcal{S})$. First, consider the case that $X_{1}=Y$ or $X_{2}=Y$. Without loss of generality, assume $X_{2}=Y$. Then, we have $\left(X_{1} \cap X_{2}, Y\right) \in \mathrm{c}_{\mathcal{H}, \mathcal{B}}(\mathcal{S})$ since $X_{1}=\left(X_{1} \cap X_{2}\right)$. Now assume $X_{1} \subset Y$ and $X_{2} \subset Y$. From $\mathcal{S}$ being $\langle\mathcal{H}, \mathcal{B}\rangle$ HI-closed, it follows that there is some $X^{\prime} \subset Y$ such that $\left(X^{\prime}, Y\right) \in \mathcal{S},\left(X_{1} \cap X_{2} \cap\right.$ $\mathcal{H}) \subseteq\left(X^{\prime} \cap \mathcal{H}\right)$, and $\left(X^{\prime} \cap \mathcal{B}\right) \subseteq\left(X_{1} \cap X_{2} \cap \mathcal{B}\right)$. From Definition 4, we conclude $\left(X_{1} \cap X_{2}, Y\right) \in \mathrm{c}_{\mathcal{H}, \mathcal{B}}(\mathcal{S})$.

We now state our main result for casting to $\mathcal{N} \mathcal{L P}$ under hyperequivalence. Its proof follows the general argumentation for establishing $(\star)$ in the beginning of this section, using the preceding results.

Theorem 6. For sets $\mathcal{H}, \mathcal{B} \subseteq$ At and a DLP P over At, there exists an NLP $Q$ over At such that $P \equiv_{\mathcal{B}}^{\mathcal{H}} Q$ iff $H E_{\mathcal{B}}^{\mathcal{H}}(P)$ is $\langle\mathcal{H}, \mathcal{B}\rangle$-HI-closed.

Example 4. Reconsider program $P_{1}$ from Example 1 and recall that there is no NLP that is strongly equivalent to $P_{1}$. We now weaken the notion of equivalence, by banning $b$ from the bodies of rules in potential context programs. So, for sets $\mathcal{H}=\{a, b, c\}$ and $\mathcal{B}=\{a, c\}$, we have $H E_{\mathcal{B}}^{\mathcal{H}}\left(P_{1}\right)=\{(b, b),(b, a b),(a b, a b),(a c, a c),(b, b c),(b c, b c)$, $(b, a b c),(a b, a b c),(a c, a b c),(b c, a b c),(a b c, a b c)\}$. As $H E_{\mathcal{B}}^{\mathcal{H}}\left(P_{1}\right)$ is $\langle\mathcal{H}, \mathcal{B}\rangle$-HI-closed, there is an NLP that is $\langle\mathcal{H}, \mathcal{B}\rangle$-equivalent to $P_{1}$, e.g., $P_{1}^{\prime}=\{a \leftarrow \operatorname{not} b ; \quad b \leftarrow$ not $a ; b \leftarrow$ not $c ; c \leftarrow$ not $b\}$. 


\subsection{Elimination of Negation}

Similarly to closure under here-intersection, we now generalise the notion of heretotality.

Definition 6. Let $\mathcal{H}, \mathcal{B}$ be sets of atoms. A set $\mathcal{S}$ of SE-interpretations is $\langle\mathcal{H}, \mathcal{B}\rangle$-heretotal if, for any pair $(X, Y) \in \mathcal{S}$ with $X \subset Y$, there is some $X^{\prime} \subset Y$ such that $\left(X^{\prime}, X^{\prime}\right) \in \mathcal{S}$ and $X=X^{\prime} \cap(\mathcal{H} \cup \mathcal{B})$.

Concerning condition $\phi_{\mathcal{B}, \mathcal{C}}^{\mathcal{H}}(\cdot)$ from the beginning of this section, its realisation in case $\mathcal{C}=\mathcal{P} \mathcal{L} \mathcal{P}$ is now given by conjoining $\langle\mathcal{H}, \mathcal{B}\rangle$-completeness and $\langle\mathcal{H}, \mathcal{B}\rangle$-here-totality.

Theorem 7. Let $\mathcal{H}, \mathcal{B} \subseteq$ At be sets of atoms and $P$ a PLP over At. Then, $H E_{\mathcal{B}}^{\mathcal{H}}(P)$ is $\langle\mathcal{H}, \mathcal{B}\rangle$-here-total.

For casting a DLP to $\mathcal{P} \mathcal{L P}$, another kind of completion is needed. We aim at mapping an $\langle\mathcal{H}, \mathcal{B}\rangle$-complete set of SE-interpretations being $\langle\mathcal{H}, \mathcal{B}\rangle$-here-total to a complete set of SE-interpretations that is here-total.

Definition 7. For any set $\mathcal{S}$ of $S E$-interpretations, let $\hat{\mathrm{c}}(\mathcal{S})$ be given by $\{(X, Y) \mid X \subseteq$ $Y,(X, X),(Y, Y) \in \mathcal{S}\}$.

Note that $\hat{c}(\cdot)$ represents the completion transformation $\tau_{\mathcal{B}, \mathcal{C}}^{\mathcal{H}}(\cdot)$ for $\mathcal{C}=\mathcal{P} \mathcal{L P}$.

The main result for casting DLPs to $\mathcal{P} \mathcal{L P}$ is formulated as follows:

Theorem 8. Let $\mathcal{H}, \mathcal{B} \subseteq$ At be sets of atoms and $P$ a DLP over At. Then, there exists a PLP $Q$ over At such that $P \equiv_{\mathcal{B}}^{\mathcal{H}} Q$ iff $H E_{\mathcal{B}}^{\mathcal{H}}(P)$ is $\langle\mathcal{H}, \mathcal{B}\rangle$-here-total.

Example 5. Again, consider program $P_{1}$ from Example 1 and recall that there is no PLP that is strongly equivalent to $P_{1}$. For sets $\mathcal{H}=\{a\}$ and $\mathcal{B}=\{a, c\}$, we have $H E_{\mathcal{B}}^{\mathcal{H}}\left(P_{1}\right)=\{(b, b),(\emptyset, a b),(a b, a b),(a c, a c)\} . H E_{\mathcal{B}}^{\mathcal{H}}\left(P_{1}\right)$ is $\langle\mathcal{H}, \mathcal{B}\rangle$-here-total, and thus there is a PLP that is $\langle\mathcal{H}, \mathcal{B}\rangle$-equivalent to $P_{1}$, e.g., $P_{1}^{\prime \prime}=\{a \vee b \leftarrow ; b \vee c \leftarrow\}$.

\subsection{Joint Elimination of Disjunction and Negation}

The characterising property of DLPs being castable to $\mathcal{H O R N}$ is independent of the body alphabet. In conjunction with $\langle\mathcal{H}, \mathcal{B}\rangle$-completeness, it constitutes $\phi_{\mathcal{B}, \mathcal{H O R N}}^{\mathcal{H}}(\cdot)$.

Definition 8. For a set $\mathcal{H}$ of atoms, a set $\mathcal{S}$ of SE-interpretations is $\mathcal{H}$-closed under there-intersection, or $\mathcal{H}$-TI-closed, if, whenever $(X, X) \in \mathcal{S}$ and $(Y, Y) \in \mathcal{S}$, there is $a(Z, Z) \in \mathcal{S}$ such that $Z \subseteq(X \cap Y)$ and $(Z \cap \mathcal{H})=(X \cap Y) \cap \mathcal{H}$.

Theorem 9. Let $\mathcal{H}, \mathcal{B} \subseteq$ At be sets of atoms and $P$ a Horn program over At. Then, $H E_{\mathcal{B}}^{\mathcal{H}}(P)$ is $\mathcal{H}$-TI-closed.

The completion transformation for casting to $\mathcal{H O R N}$, defined next, reuses the completion $\hat{c}(\cdot)$ from Definition 7 . However, a refinement is necessary for guaranteeing closure under there-intersection of the respective mapping.

Definition 9. Let $\mathcal{H} \subseteq$ At be a set of atoms. For every set $\mathcal{S}$ of SE-interpretations, let $\tilde{\mathrm{c}}_{\mathcal{H}}(\mathcal{S})$ be given by $\hat{\mathrm{c}}\left(\left\{\left(Z^{\prime}, Z^{\prime}\right) \mid(Z, Z) \in \mathcal{S}, Z \subseteq Z^{\prime} \subseteq A t,\left(Z^{\prime} \cap \mathcal{H}\right)=(Z \cap \mathcal{H})\right\}\right)$. 
As in the previous cases, $\tilde{c}_{\mathcal{H}}(\cdot)$ represents $\tau_{\mathcal{B}, \mathcal{C}}^{\mathcal{H}}(\cdot)$ for $\mathcal{C}=\mathcal{H O} \mathcal{R N}$. As well, in Section 5 we will summarise the role of the completion transformations $\mathcal{C}_{\mathcal{H}, \mathcal{B}}(\cdot), \hat{\mathrm{c}}(\cdot)$, and $\tilde{\mathrm{c}}_{\mathcal{H}}(\cdot)$ for computing a casting of a given program.

Theorem 10. Let $\mathcal{H}, \mathcal{B} \subseteq$ At be sets of atoms and $P$ a DLP over At. Then, there is a Horn program $Q$ over At with $P \equiv_{\mathcal{B}}^{\mathcal{H}} Q$ iff $H E_{\mathcal{B}}^{\mathcal{H}}(P)$ is $\langle\mathcal{H}, \mathcal{B}\rangle$-here-total and $\mathcal{H}$-TI-closed.

Example 6. Consider program $P_{2}$ from Example 1 and recall from Example 3 that there is no Horn program strongly equivalent to $P_{2}$. For sets $\mathcal{H}=\{a, c\}$ and $\mathcal{B}=$ $\{a, b, c\}$, we have $H E_{\mathcal{B}}^{\mathcal{H}}\left(P_{1}\right)=\{(\emptyset, \emptyset),(\emptyset, a b),(a b, a b),(\emptyset, b c),(b c, b c)\} . H E_{\mathcal{B}}^{\mathcal{H}}\left(P_{2}\right)$ is $\langle\mathcal{H}, \mathcal{B}\rangle$-here-total and $\langle\mathcal{H}, \mathcal{B}\rangle$-TI-closed. Thus, there is a Horn program that is $\langle\mathcal{H}, \mathcal{B}\rangle$ equivalent to $P_{2}$, e.g., $P_{2}^{\prime}=\{b \leftarrow a ; b \leftarrow c ; \leftarrow a, c\}$.

\subsection{Special Cases}

We briefly discuss our results with respect to important corner cases of hyperequivalence. Naturally, for strong equivalence, the introduced characterisations reduce to the notions presented in Section 3. Hence, we provided proper generalisations of the concepts known for this special case. For identical head and body alphabets, hyperequivalence reduces to relativised strong equivalence as introduced by Woltran [13]. Interestingly, $\langle A, A\rangle$-closure under here-intersection reduces to ordinary closure under here-intersection for $\langle A, A\rangle$-well-defined sets of SE-interpretations. Consequently, for relativised strong equivalence, we get the following refinement of Theorem 6 :

Theorem 11. Let $A \subseteq A t$ be a set of atoms and $P$ a DLP over At. Then, there exists an NLP $Q$ over At such that $P \equiv_{A}^{A} Q$ iff $H E_{A}^{A}(P)$ is HI-closed.

\section{Computational Aspects of Program Casting}

In this section, we first summarise how program castings can be computed, and afterwards we discuss the complexity of casting under hyperequivalence.

Let $\mathcal{H}, \mathcal{B} \subseteq A t$ be sets of atoms. For obtaining an $\langle\mathcal{H}, \mathcal{B}\rangle$-casting of a given DLP $P$ over $A t$ to class $\mathcal{C}$ with (i) $\mathcal{C}=\mathcal{N} \mathcal{L} \mathcal{P}$, (ii) $\mathcal{C}=\mathcal{P} \mathcal{L} \mathcal{P}$, or (iii) $\mathcal{C}=\mathcal{H O} \mathcal{R} \mathcal{N}$, respectively, where $P$ is castable to $\mathcal{C}$ under $\langle\mathcal{H}, \mathcal{B}\rangle$-equivalence, one can proceed as follows:

1. Compute $H E_{\mathcal{B}}^{\mathcal{H}}(P)$.

2. Depending on the class $\mathcal{C}$ of programs, compute (i) $\mathcal{S}=\mathrm{c}_{\mathcal{H}, \mathcal{B}}\left(H E_{\mathcal{B}}^{\mathcal{H}}(P)\right)$, (ii) $\mathcal{S}=$ $\hat{\mathrm{c}}\left(H E_{\mathcal{B}}^{\mathcal{H}}(P)\right)$, or (iii) $\mathcal{S}=\tilde{\mathrm{c}}_{\mathcal{H}}\left(H E_{\mathcal{B}}^{\mathcal{H}}(P)\right)$.

3. Compute the canonical program $\mathrm{CP}_{\mathcal{S}}$ for $\mathcal{S}$.

4. Apply techniques to remove (i) disjunctions, (ii) negations, or (iii) negations and disjunctions from $\mathrm{CP}_{\mathcal{S}}$, preserving strong equivalence, as discussed in Section 3.

For analysing the complexity of $\operatorname{CAST}(\mathcal{C})$ for $\mathcal{C} \in\{\mathcal{N} \mathcal{L P}, \mathcal{P} \mathcal{L P}, \mathcal{H O} \mathcal{R} \mathcal{N}\}$, we need the following membership result for $\langle\mathcal{H}, \mathcal{B}\rangle$-model checking.

Theorem 12. The problem of deciding whether $(X, Y) \in H E_{\mathcal{B}}^{\mathcal{H}}(Q)$, for given $X, Y, \mathcal{H}$, $\mathcal{B} \subseteq$ At and DLP $Q$ over At, is in $\Delta_{2}^{P}$. 
The following relationship between $\langle\mathcal{H}, \mathcal{B}\rangle$-models and SE-models is essential for efficiently checking whether a DLP can be replaced by a hyperequivalent NLP.

Lemma 6. Let $X, Y, \mathcal{H}, \mathcal{B} \subseteq$ At be sets of atoms, and $P$ a DLP over At such that $X \subseteq(\mathcal{H} \cup \mathcal{B})$. If $(Y, Y) \in \bar{H} E_{\mathcal{B}}^{\mathcal{H}}(P)$, then the following statements are equivalent:

- there is some $X^{\prime} \subset Y$ with $\left(X^{\prime}, Y\right) \in H E_{\mathcal{B}}^{\mathcal{H}}(P),(X \cap \mathcal{H}) \subseteq\left(X^{\prime} \cap \mathcal{H}\right)$, and $\left(X^{\prime} \cap \mathcal{B}\right) \subseteq(X \cap \mathcal{B})$;

- there is some $X^{\prime \prime} \subset Y$ with $\left(X^{\prime \prime}, Y\right) \in S E(P),(X \cap \mathcal{H}) \subseteq\left(X^{\prime \prime} \cap \mathcal{H}\right)$, and $\left(X^{\prime \prime} \cap \mathcal{B}\right) \subseteq(X \cap \mathcal{B})$.

Theorem 13. Deciding $\operatorname{CAST}(\mathcal{N} \mathcal{L} \mathcal{P})$ is $\Pi_{2}^{P}$-complete. Moreover, the problem remains $\Pi_{2}^{P}$-hard if we restrict it to instances where $\mathcal{H}=\mathcal{B}$ holds.

Proof (Sketch). For membership, we show that the complementary problem, i.e., deciding whether $H E_{\mathcal{B}}^{\mathcal{H}}(P)$ is not $\langle\mathcal{H}, \mathcal{B}\rangle$-HI-closed, is in $\Sigma_{2}^{P}$. Note that $H E_{\mathcal{B}}^{\mathcal{H}}(Q)$ is $\langle\mathcal{H}, \mathcal{B}\rangle$-HI-closed iff $H E_{\mathcal{B}}^{\mathcal{H}}(Q) \cap(\operatorname{atm}(Q) \times \operatorname{atm}(Q))$ is $\langle\mathcal{H}, \mathcal{B}\rangle$-HI-closed. We can nondeterministically guess some $X_{1}, X_{2}, Y \subseteq \operatorname{atm}(Q)$. By Theorem 12, a polynomial number of NP-oracle calls suffice to decide whether $\left(X_{1}, Y\right) \in H E_{\mathcal{B}}^{\mathcal{H}}(Q)$ and $\left(X_{2}, Y\right) \in H E_{\mathcal{B}}^{\mathcal{H}}(Q)$. As a consequence of Lemma 6, an NP-oracle can be used to check whether there is no $X^{\prime} \subset Y$ with $\left(X^{\prime}, Y\right) \in H E_{\mathcal{B}}^{\mathcal{H}}(Q),\left(X_{1} \cap X_{2}\right) \cap \mathcal{H} \subseteq\left(X^{\prime} \cap \mathcal{H}\right)$, and $\left(X^{\prime} \cap \mathcal{B}\right) \subseteq\left(X_{1} \cap X_{2}\right) \cap \mathcal{B}$. Thus, a nondeterministic algorithm with access to an NP-oracle solves the complementary problem in polynomial time.

We show hardness by a reducing the problem of deciding the truth of a quantified Boolean formula $(\mathrm{QBF})$ to checking whether $H E_{\mathcal{B}}^{\mathcal{H}}(P)$ is not $\langle\mathcal{H}, \mathcal{B}\rangle$-HI-closed. Consider a QBF $\Phi=\exists K \forall L \delta_{1} \vee \cdots \vee \delta_{r}$, where each $\delta_{i}$ is a conjunction of literals over $K \cup L$. We assume that $K \neq \emptyset$ and $L \neq \emptyset$. For every $x \in K \cup L$, we denote by $\bar{x}$ a globally new atom not appearing anywhere in $\phi$. Given a set $X$ of atoms, we define $\bar{X}=\{\bar{x} \mid x \in X\}$. Finally, for each conjunction $\delta=a_{1} \wedge \cdots \wedge a_{h} \wedge \neg a_{h+1} \wedge \neg a_{n}$ of literals, we denote by $\delta^{\dagger}$ the sequence $a_{1}, \ldots, a_{h}, \overline{a_{h+1}}, \ldots, \overline{a_{n}}$ and define program $P_{\Phi}$, using further new atoms $a, b, w$, as follows.

$$
\begin{aligned}
P_{\Phi}= & \{k \leftarrow \operatorname{not} \bar{k} ; \bar{k} \leftarrow \text { not } k ; \leftarrow k, \bar{k} \mid k \in K\} \cup \\
& \{l \vee \bar{l} \leftarrow ; w \leftarrow l, \bar{l} ; l \leftarrow w ; \bar{l} \leftarrow w ; \mid l \in L\} \cup \\
& \left\{a \vee b \leftarrow \delta_{i}^{\dagger} ; w \leftarrow \delta_{i}^{\dagger} ; \mid 1 \leq i \leq r\right\} .
\end{aligned}
$$

For $A=K \cup \bar{K} \cup\{a, b\}$, it can be shown that $\Phi$ is true iff $H E_{A}^{A}\left(P_{\Phi}\right)$ is not HI-closed, and therefore, by Theorem 11, not $\langle A, A\rangle$-HI-closed. Since deciding the truth of a QBF of form $\Phi$ is $\Sigma_{2}^{P}$-hard, the assertion follows from that.

It was shown [8] that there is no rewriting $f$ from DLPs to NLPs such that $P \equiv{ }_{\emptyset}^{A t}$ $f(P)$, where $f(P)$ is polynomial in the size of $P$, for every program $P$, unless the polynomial hierarchy $(\mathrm{PH})$ collapses. Hence, there is also no polynomial rewriting from DLPs to NLPs for the general case of hyperequivalence, unless the PH collapses.

It turns out that checking whether a DLP can be replaced by a hyperequivalent PLP is computationally more expensive than deciding whether there is a corresponding NLP.

Theorem 14. Deciding $\mathrm{CAST}(\mathcal{P} \mathcal{L} \mathcal{P})$ is $\Pi_{3}^{P}$-complete. Moreover, the problem remains $\Pi_{3}^{P}$-hard if we restrict it to instances where $\mathcal{B} \subseteq \mathcal{H}$ holds. 
Proof (Sketch). We only show the hardness part. Consider a QBF $\Phi=\exists K \forall L \exists M \phi$, where $\phi$ is a formula in CNF over the set $K \cup L \cup M$ of atoms. We assume that $K \neq \emptyset$, $L \neq \emptyset$, and $M \neq \emptyset$. As before, for every $x \in K \cup L \cup M$, we denote by $\bar{x}$ a globally new atom not appearing anywhere in $\phi$, and we define $\bar{X}=\{\bar{x} \mid x \in X\}$ for every set $X$ of atoms. Finally, for each clause $\gamma=x_{1} \vee \cdots \vee x_{k} \vee \neg x_{k+1} \vee \cdots \vee \neg x_{n}$, we denote by $\gamma^{\ddagger}$ the sequence $\bar{x}_{1}, \ldots, \bar{x}_{k}, x_{k+1}, \ldots, x_{n}$, and define a program $P_{\Phi}$, using a further new atom $w$, as follows:

$$
\begin{aligned}
P_{\Phi}= & \{j \vee \bar{j} \leftarrow ; o \leftarrow j, \bar{j} ; \bar{o} \leftarrow j, \bar{j} \mid j \in K \cup L, o \in K \cup L \cup M\} \cup \\
& \{m \vee \bar{m} \leftarrow ; w \leftarrow m, \bar{m} ; m \leftarrow w ; \bar{m} \leftarrow w \mid m \in M\} \cup \\
& \left\{w \leftarrow \gamma^{\ddagger} \mid \text { for each clause } \gamma \text { in } \phi\right\} \cup\{w \leftarrow \text { not } w\} .
\end{aligned}
$$

For $\mathcal{B} \subseteq \mathcal{H}=K \cup \bar{K}$, it can be shown that $\Phi$ is true iff $H E_{\mathcal{B}}^{\mathcal{H}}\left(P_{\Phi}\right)$ is not $\langle\mathcal{H}, \mathcal{B}\rangle$-heretotal. As deciding the truth of a QBF of form $\Phi$ is $\Sigma_{3}^{P}$-hard, the assertion follows.

Theorem 15. Given set At of atoms, there is no rewriting $f: \mathcal{D} \mathcal{L} \mathcal{P} \times A t \times A t \rightarrow \mathcal{P} \mathcal{L} \mathcal{P}$ such that, for given sets $\mathcal{H}, \mathcal{B} \subseteq$ At of atoms and a $D L P P$ that is castable to $\mathcal{P} \mathcal{L P}$, $P \equiv{ }_{\mathcal{B}}^{\mathcal{H}} f(P, \mathcal{H}, \mathcal{B})$ and $f(P, \mathcal{H}, \mathcal{B})$ is polynomial in the size of $P$, unless the polynomial hierarchy collapses. The result also holds under the restriction $\mathcal{B} \subseteq \mathcal{H}$.

Proof. Let $\mathcal{H}, \mathcal{B}$ be sets of atoms such that $\mathcal{B} \subseteq \mathcal{H}$. Assume that a polynomial-size rewriting $f$ of the described kind exists. We can guess a PLP $P^{\prime}$, polynomial in the size of $P$, in nondeterministic polynomial time. Since checking $P^{\prime} \equiv_{\mathcal{B}}^{\mathcal{H}} P$ is in $\Pi_{2}^{P}$ [5], a nondeterministic algorithm with access to a $\Sigma_{2}^{P}$-oracle can decide $\operatorname{CAST}(\mathcal{P} \mathcal{L P})$. This yields membership of $\operatorname{CAST}(\mathcal{P L P})$ in $\Sigma_{3}^{P}$, which is a contradiction to the $\Pi_{3}^{P}$-hardness of the problem, unless the polynomial hierarchy collapses.

Finally, we have the following result for the Horn case:

Theorem 16. Deciding $\operatorname{CAST}(\mathcal{H O} \mathcal{R N})$ is in $\Pi_{3}^{P}$.

\section{Discussion}

We studied casting of disjunctive answer-set programs under hyperequivalence and provided necessary and sufficient semantical conditions, deciding for a program $P$, whether there exists a program $Q$ of a given syntactic subclass of DLPs which is hyperequivalent to $P$. Moreover, we provided methods for constructing such a $Q$ and studied the complexity of deciding whether casting can be applied. Here, an open issue is determining exact complexity bounds for $\operatorname{CAST}(\mathcal{H O R N})$.

Other open issues concern the canonical program we used to obtain our results. First of all, it would be valuable to have canonical programs which are "class sensitive", i.e., given a set $\mathcal{S}$ of SE-interpretations, the associated canonical program is one which is exactly in that class which is characterised by $\mathcal{S}$. This would circumvent the fourth and last step of our program casting algorithm as sketched in Section 5. Secondly, having a canonical program directly for HE-models rather than for SE-models would further simplify the task. 
An interesting application of casting under hyperequivalence is modular programming, when modules are to be replaced by syntactically simpler programs. As it is typically known which atoms are allowed to occur in the heads and bodies of rules in context modules, adequate casting can be applied even when impossible under strong equivalence. Furthermore, casting under hyperequivalence gives insight into when a syntactical class is intrinsically needed, or contrarily, when connectives like disjunction and negation are dispensable. By varying the hyperequivalence parameters, atoms which are responsible for the need of a connective can be identified. Based on the notions developed, in a next step, properties can be extracted which allow for constructing methods for determining the strongest equivalence notion under which casting is possible. That is, for a given program $P$ and syntactic class $\mathcal{C}$, find $\mathcal{H}$ and $\mathcal{B}$ such that $P$ is castable to $\mathcal{C}$ under $\langle\mathcal{H}, \mathcal{B}\rangle$-equivalence and $\mathcal{H}$ and $\mathcal{B}$ satisfy some optimality condition.

Another crucial matter for future work is research into casting with equivalence under projection [12], where answer sets need to be identical only on selected atoms. Usually, one is interested in the behaviour of a program with respect to distinguished input and output atoms. By varying parameters $\mathcal{H}$ and $\mathcal{B},\langle\mathcal{H}, \mathcal{B}\rangle$-equivalence allows for specifying the input part. Projection, on the other hand, is needed for determining output atoms. Once conditions for casting with projections are defined, it can be decided whether the respective task allows for replacing a program by a simpler one.

\section{References}

1. Baral, C.: Knowledge Representation, Reasoning and Declarative Problem Solving. Cambridge University Press (2003)

2. Gelfond, M., Lifschitz, V.: Classical negation in logic programs and disjunctive databases. New Generation Computing 9 (1991) 365-385

3. Lifschitz, V., Pearce, D., Valverde, A.: Strongly equivalent logic programs. ACM Transactions on Computational Logic 2(4) (2001) 526-541

4. Eiter, T., Fink, M.: Uniform equivalence of logic programs under the stable model semantics. In Proc. ICLP'03. LNCS, Springer (2003) 224-238

5. Woltran, S.: A common view on strong, uniform, and other notions of equivalence in answerset programming. Theory and Practice of Logic Programming 8(2) (2008) 217-234

6. Truszczyński, M., Woltran, S.: Relativized hyperequivalence of logic programs for modular programming. In Proc. ICLP'08. LNCS, Springer (2008)

7. Eiter, T., Fink, M., Tompits, H., Woltran, S.: Simplifying logic programs under uniform and strong equivalence. In Proc. LPNMR'07. LNCS, Springer (2004) 87-99

8. Eiter, T., Fink, M., Tompits, H., Woltran, S.: On eliminating disjunctions in stable logic programming. In Proc. KR'04, AAAI Press (2004) 447-458

9. Turner, H.: Strong equivalence made easy: Nested expressions and weight constraints. Theory and Practice of Logic Programming 3(4-5) (2003) 602-622

10. Heyting, A.: Die formalen Regeln der intuitionistischen Logik. Sitzungsberichte, physik.math. Klasse, preußische Akademie der Wissenschaften (1930)

11. Gödel, K.: Zum intuitionistischen Aussagenkalkül. Anzeiger Akademie der Wissenschaften in Wien, math.-naturwiss. Klasse 69 (1932) 65-66

12. Eiter, T., Tompits, H., Woltran, S.: On solution correspondences in answer set programming. In Proc. IJCAI'05. (2005) 97-102

13. Woltran, S.: Characterizations for relativized notions of equivalence in answer set programming. In Proc. JELIA'04. LNCS, Springer (2004) 161-173 